A comparative approach for the characterization of a pneumatic piston gauge up to $8 \mathrm{MPa}$ using finite element calculations

This article has been downloaded from IOPscience. Please scroll down to see the full text article.

2011 Meas. Sci. Technol. 22025103

(http://iopscience.iop.org/0957-0233/22/2/025103)

View the table of contents for this issue, or go to the journal homepage for more

Download details:

IP Address: 59.144.72.1

The article was downloaded on 23/08/2011 at 10:03

Please note that terms and conditions apply. 


\title{
A comparative approach for the characterization of a pneumatic piston gauge up to 8 MPa using finite element calculations
}

\author{
Sugandha Dogra, Jasveer Singh, Abhishek Lodh, Nita Dilawar Sharma \\ and A K Bandyopadhyay
}

Pressure and Vacuum Standards, National Physical Laboratory, New Delhi 110012, India

E-mail: ndilawar@mail.nplindia.org

Received 19 May 2010, in final form 12 November 2010

Published 23 December 2010

Online at stacks.iop.org/MST/22/025103

\begin{abstract}
This paper reports the behavior of a well-characterized pneumatic piston gauge in the pressure range up to $8 \mathrm{MPa}$ through simulation using finite element method (FEM). Experimentally, the effective area of this piston gauge has been estimated by cross-floating to obtain $A_{0}$ and $\lambda$. The FEM technique addresses this problem through simulation and optimization with standard commercial software (ANSYS) where the material properties of the piston and cylinder, dimensional measurements, etc are used as the input parameters. The simulation provides the effective area $A_{p}$ as a function of pressure in the free deformation mode. From these data, one can estimate $A_{p}$ versus pressure and thereby $A_{o}$ and $\lambda$. Further, we have carried out a similar theoretical calculation of $A_{p}$ using the conventional method involving the Dadson's as well as Johnson-Newhall equations. A comparison of these results with the experimental results has been carried out.
\end{abstract}

Keywords: pressure balance, pressure metrology, finite element analysis

(Some figures in this article are in colour only in the electronic version)

\section{Introduction}

In the pneumatic pressure region up to $8 \mathrm{MPa}$, although the pressure-induced distortion of the effective area as a function of pressure $A_{p}$ is notably smaller than the hydraulic pressure region above $100 \mathrm{MPa}$, it is still considerably high and contributes significantly to the overall uncertainty of the measurement in pressure. For this reason the evaluation of $A_{p}$ of a piston-cylinder assembly has been carried out both experimentally and theoretically. A number of different theoretical techniques have been employed in the past for these evaluations so that the difference between them is reasonably reduced. To derive the effective area of a particular pistoncylinder combination by numerical methods, one needs to be able to calculate force due to applied line pressure acting on the cross-sectional area of the lower end of the piston, allowing for the change of cross-section at that end due to combined effects of axial and radial compression as well as the vertical component of force due to the pressure acting on the sides of the piston.

The basic problems due to which theoretical estimations suffer are (1) the geometric irregularities of the surface area in the clearance between the piston-cylinder assembly, (2) the vertical force distribution on the piston-cylinder under compression, (3) the pressure gradient in the clearance length between the piston and the cylinder [1]. All the components are interdependent and complex in nature, and very few available methods provide solution. It is for this reason a number of quite different techniques have been reported to be used, and among them application of the finite element method (FEM) has been quite significant and important $[2,3]$. The complexity of the geometry and the boundary conditions makes the FEM more reasonable in solving mechanical problems. Pressure balance modeling traditionally considered that undistorted piston and 


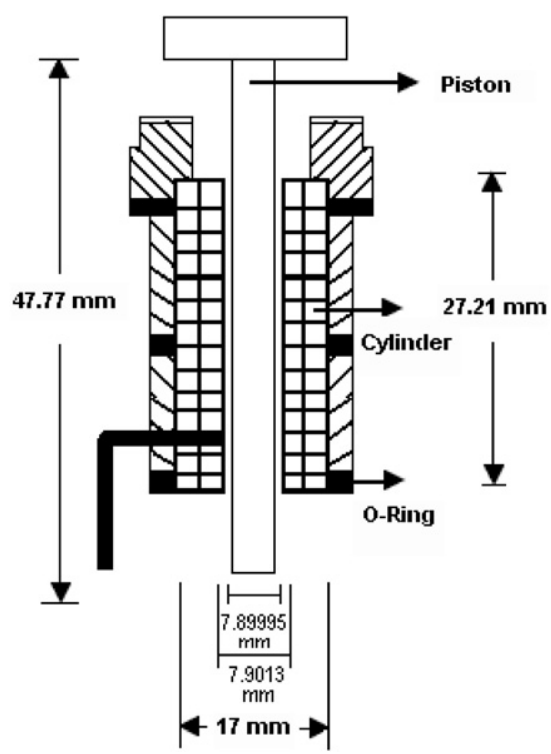

Figure 1. Schematic of NPL-8A with dimensions.

cylinder are perfectly round and perfectly straight. Although attempts have been made to take into account real shapes of pistons and cylinders, these have always been based on deriving some averaged profile for piston and cylinder that is then used as the basis for the axisymmetric model.

In addition, the elastic theory by Dadson and Peggs $[4,5]$ has been quite successful. In this paper, we will report a brief study of a piston gauge in pneumatic pressure range up to $8 \mathrm{MPa}$ using the above-mentioned FE analysis using ANSYS and compare the results to those obtained from Dadson's theory as well as from the cross-float experiments. The simulation has been done using the simplest 2D modeling of the piston-cylinder assembly.

This simulation using FEM involves making an initial approximation at pressure distribution along the gap, the simplest approach being to assume initial linear pressure distribution, and use this pressure distribution as input to solution of structural deformation problem. Simplified equations require one to know the radius of the piston and inner radius of the cylinder, which have been measured at Dimension Measurements Laboratory at NPLI.

\section{Experimental details}

\subsection{Description of the NPL-8A piston gauge}

NPLI-8A is an oil-lubricated simple piston-cylinder system capable of measuring the full-scale pneumatic pressure up to $8 \mathrm{MPa}$. It is a DH-Budenberg made model 5502 with piston-cylinder serial no 2943. The diagrammatic view of the NPL-8A piston-cylinder assembly with important geometrical characteristics is shown in figure 1 . The relative motion between the piston and the cylinder is obtained by a motor mounted away from the piston-cylinder assembly. The cylinder sits in a cavity and is held by a retaining nut at the top. Although not directly, the bottom of the cylinder still experiences the applied pressure.
Table 1. Input parameters for the basic FEA model: geometry and material properties.

\begin{tabular}{ll}
\hline Geometry (mm) & \\
Piston radius & 3.949975 \\
Initial clearance & 0.000675 \\
Cylinder radius (inner) & 3.95065 \\
Cylinder radius (outer) & 8.5 \\
Cylinder length & 27.21 \\
Piston length & 47.77 \\
Engagement length & 27.21 \\
Material properties & \\
$\quad$ Piston & \\
Young's modulus & $630 \mathrm{GPa}$ \\
Poisson's ratio & 0.218 \\
Cylinder & \\
Young's modulus & $630 \mathrm{GPa}$ \\
Poisson's ratio & 0.218 \\
\hline
\end{tabular}

This pressure balance has participated in many intercomparisons and key comparisons [6-8]. The dimensions of the NPLI-8A piston-cylinder assembly have been estimated at the Dimension Metrology division at NPLI. A number of measurements are carried out at three different heights and at different angles. The mean measured diameters of the piston and cylinder are 7.89995 and $7.9013 \mathrm{~mm}$ respectively. The expanded uncertainty in measurement is $\pm 0.7 \mu \mathrm{m}$ at coverage factor $k=2$. However, since only the radii values for the piston and cylinder are being used for computations, the expanded uncertainty would be approximately $\pm 0.5 \mu \mathrm{m}$ at $k=2$ [9]. The piston and cylinder have radii of 3.949975 and $3.95065 \mathrm{~mm}$ respectively, and their corresponding areas are $4.9016079 \times 10^{-5}$ and $4.9032833 \times 10^{-5} \mathrm{~m}^{2}$ respectively. Therefore, the effective area $A_{o}$ (initial area) is $4.902445586 \times$ $10^{-5} \mathrm{~m}^{2}$, which is the arithmetic mean between the area of the piston and that of the cylinder. The initial clearance between the piston and cylinder is $h_{o}=0.675 \mu \mathrm{m}$ along the engagement length. The nominal outer diameter of the cylinder is $17 \mathrm{~mm}$. Both the piston and cylinder are made of tungsten carbide with Young's modulus E as $630 \mathrm{GPa}$ and Poisson's ratio as $0.218^{1}$. The values of Young's modulus and Poisson ratio have been taken from the manufacturer's data. Table 1 lists the input parameters used for FEM simulations.

\subsection{Reference standard}

The reference standard is designated as NPLI-4 and is a dead weight tester, manufactured by Ruska Inc., Texas, USA, model no 2465 , piston serial no V-607, with a nominal effective area of $8.4 \mathrm{~mm}^{2}$. Its piston-cylinder assembly is capable of measuring the pressure over a range of $0.2-4 \mathrm{MPa}$. This was used as a reference standard in the APMP.M.P-k1c during 1999-2001 [6].

In NPLI-4, both the piston and the cylinder are made of cemented tungsten carbide with $6 \%$ Co. The relative motion of the gas-lubricated piston is obtained by rotating the cylinder around the piston to relieve friction. The zero pressure effective area $\left[A_{o}\right]$ and pressure distortion coefficient $[\lambda]$ for this standard are traceable to the Ultrasonic

\footnotetext{
1 Operation Manual DH-Budenberg Dead Weight Tester Model 5502.
} 
Interferometer Manometer at NPL through a continuous unbroken chain. The zero pressure effective area at $20{ }^{\circ} \mathrm{C}$ was found to be $8.392198 \times 10^{-6} \mathrm{~m}^{2}$ and the pressure distortion coefficient is $4.88 \times 10^{-6} \mathrm{MPa}^{-1}$ [10]. NPLI-4 has participated in bilateral comparison with PTB Germany in 1988 and with NIST, USA, in bilateral comparison APMP.SIM.M.P-k1c [7]. The relative stability of $A_{o}$ during the span of the last 20 years has remained within $4 \times 10^{-6}$. The expanded uncertainty of NPLI-4 in pressure has been recently re-established and has been estimated to be $22 \times 10^{-6}$ at $k=$ 2 [11].

\subsection{Experimental procedure}

The two units were intercompared using the cross-float technique for the piston gauges. All measurements were carried out in a controlled environment with the ambient maintained at a temperature of $23 \pm 1{ }^{\circ} \mathrm{C}$ and the relative humidity at $50 \pm 5 \%$. The piston gauges were mounted on a heavy stainless steel base to minimize vibrations and magnetic effects. Purified nitrogen gas was used as the pressurizing medium. About 15 min of time was allowed for changes in pressure to stabilize and bring the system into equilibrium. The pressure reference level of the two piston gauges was identified as the mid-stroke position when both pistons were in equilibrium position. A pressure head correction term was applied to compensate for the difference in the reference levels of the pistons. The observations were taken at nominal pressure values of $0.4,0.8,1.2,1.6,2.0,2.4,2.8,3.2,3.6$ and $4 \mathrm{MPa}$ and three increasing as well as three decreasing pressure cycles were completed.

\section{Estimation of the effective area $A_{o}$}

The experimental zero pressure effective area was obtained by conventional cross-floating of the two piston gauges. Although the working temperature was controlled around $23{ }^{\circ} \mathrm{C}$, the calculations were done at $20^{\circ} \mathrm{C}$. Hence, the effective area of the test standard for each observation at $20^{\circ} \mathrm{C}$ was calculated using the equation

$$
A_{p}=\frac{\sum_{i} m_{i} g_{i}\left(1-\rho_{\mathrm{air}} / \rho_{m}\right)}{P_{1}\left[1+\left(\alpha_{p}+\alpha_{c}\right)\left(T-T_{0}\right)\right]},
$$

where $P_{1}$ is the standard generated pressure at the bottom of the piston, $m_{i}$ is the mass of the weights, $\rho_{\text {air }}$ and $\rho_{m}$ are the densities of air and mass respectively, $\alpha_{p}$ and $\alpha_{c}$ are the thermal expansion coefficients of the piston and cylinder respectively, $g_{i}$ is the local acceleration due to gravity, $T$ is the temperature of the piston-cylinder assembly and $T_{0}$ is the temperature at which $A_{0}$ is specified. The average value of the effective area at each pressure point from $n=6$ observations is

$$
A_{p, \text { av }}=\frac{\sum_{k=1}^{n} A_{p, k}}{n} .
$$

The effective area data from the test standard at each observation have been fitted by least-squares regression to the linear distortion model:

$$
A_{p}=a+b P_{1}=A_{o}\left(1+\lambda P_{1}\right)
$$

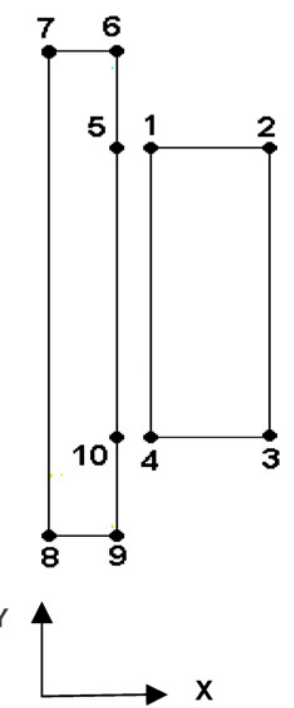

Figure 2. Model of the piston-cylinder assembly with key points used for calculations.

to determine the value of zero pressure effective area $A_{0}$, and the distortion coefficient $\lambda$. Hence, $a=A_{0}$ and $b=A_{o} \lambda$. The uncertainty in the effective area is computed using the specified ISO and NABL guidelines [12].

\section{Modeling of piston and cylinder for elastic distortion calculation}

As already mentioned, the elastic distortion of the piston and cylinder was determined by simulation using ANSYS FEM program. Both the effective area after distortion and distortion coefficient must be known with accuracy and should be comparable with the theoretically calculated value of the distortion. In order to make a consistency check it was decided to determine the zero pressure effective area of the NPLI-8A from both theoretical calculations using Dadson's equations [4], FEM simulation, and by extrapolating the graph of the experimental effective area versus pressure. Also, as already mentioned, the effective area at atmospheric pressure is determined by taking into consideration the diametrical measurements from our dimensional metrology laboratory. As already mentioned, a number of measurements were carried out at three different heights at different angles, and the mean values of these results have been taken.

Prior to computation, a model of the piston-cylinder assembly was designed. The piston-cylinder assembly is taken as a 2D model [2, 3, 13] assuming axial symmetry as shown in figure 2. Assuming point no 8 in figure 2 as the origin, the coordinates of the other key points were calculated. Accordingly, the $x-y$ coordinates of the key points nos 5, 10 of the piston and 1, 4 of the cylinder are (3.949 975, $37.49),(3.949975,10.28)$ and $(3.950650,37.49)$, (3.950 650, 10.28) respectively. These points are summarized in table 2 . The engagement length is $27.21 \mathrm{~mm}$, which is the difference between the $x$ coordinates of key point nos 5 and 10 or 1 and 4.

The meshed figure of piston-cylinder assembly with smart size 4 is shown in figure 3(a) and its zoomed view is shown in 
Table 2. The other major $x-y$ coordinates of the NPL-8A piston-cylinder assembly estimated as per the dimensions given in table 1.

\begin{tabular}{lll}
\hline Key points & $x(\mathrm{~mm})$ & $y(\mathrm{~mm})$ \\
\hline Cylinder & & \\
1 & 3.9506 & 37.49 \\
2 & 8.5 & 37.49 \\
3 & 8.5 & 10.28 \\
4 & 3.9506 & 10.28 \\
Piston & & \\
5 & 3.949975 & 37.49 \\
6 & 3.949975 & 47.77 \\
7 & 0 & 47.77 \\
8 & 0 & 0 \\
9 & 3.949975 & 0 \\
10 & 3.949975 & 10.28 \\
\hline
\end{tabular}

figure $3(b)$. As can be seen from the figures, for the estimation of strains and distortions along the engagement length where the applied pressure component acts, the concentration of nodes is much larger. After meshing, there was a total of 578 nodes created along the engagement length of $27.21 \mathrm{~mm}$ and a total number of 6925 ( 8 node) quadrilateral elements in the piston-cylinder assembly. After creating the areas determined by the main key points, we have demarcated constant pressure on lines 8-9, 9-10 and 3-4. A linearly varying pressure is applied along the engagement length (lines 5-10 and 1-4 of the piston and cylinder respectively). The FEM calculations were carried out for pressures of $1 \mathrm{MPa}$, 1.5 MPa, 2 MPa, 2.5 MPa, 3 MPa, 3.5 MPa, 4 MPa, 4.5 MPa, $5 \mathrm{MPa}, 5.5 \mathrm{MPa}, 6 \mathrm{MPa}, 6.5 \mathrm{MPa}, 7 \mathrm{MPa}, 7.5 \mathrm{MPa}$, and $8 \mathrm{MPa}$. Lines $1-2$ of the cylinder and 6-7 of the piston have been constrained to $y$-direction to restrict the movement of piston-cylinder assembly [14].

For comparison purposes, the calculations were repeated for mesh sizes 3 and 5. In the former case 771 nodes were created along the engagement length, while for the latter case 357 nodes were created.

\section{Elastic distortion theory}

For simple piston-cylinder geometry the effective area under pressure is given by equation (3). As already mentioned, the NPLI-8A, with pressure range $8 \mathrm{MPa}$ pneumatic pressure unit with a nominal effective area of $4.902445586 \times 10^{-5} \mathrm{~m}^{2}$ and piston-cylinder made of tungsten carbide operating in free deformation mode (FDM) was selected as the object for our calculations.

\subsection{Calculation details}

In the present work, $A_{p}$ as a function of pressure was calculated using two approaches, theoretical and simulated. Further, the distortion coefficient and the zero pressure effective area were also calculated. Theoretical calculations were carried out using Dadson's equations [4] and Johnson/Newhall equations [15], and the simulations were carried out using the commercially available ANSYS software along with Dadson's equations. The results of both the calculations were

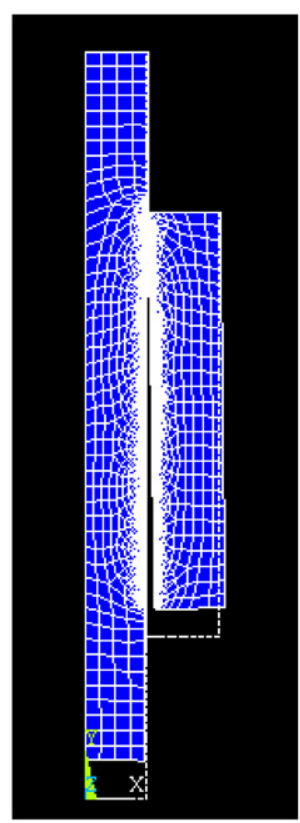

(a)

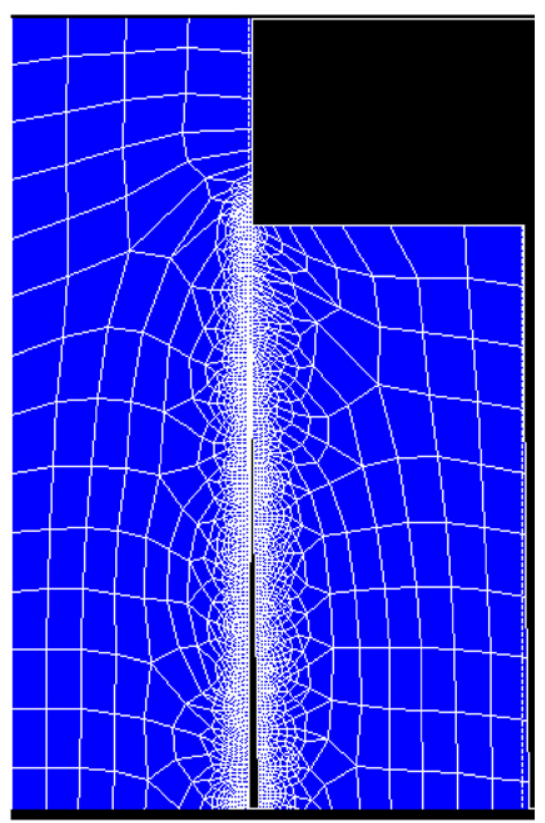

(b)
Figure 3. Meshed figure of NPL-8A pc assembly showing $(a)$ deformed shape of the piston-cylinder assembly, $(b)$ zoomed meshed figure of the engagement length.

further compared to each other as well as the results of our experimental cross-floating.

In both the calculations, $A_{p}$ can be calculated using Dadson's theory [4] and is derived as

$$
\begin{aligned}
A_{p}= & \pi r_{p_{o}}^{2}\left[1+\frac{h_{o}}{r_{p_{o}}}-\frac{1}{r_{p_{o}}\left(P_{1}-P_{2}\right)}\right. \\
& \left.\times \int_{0}^{l}\left[\{u(x)+U(x)\} \frac{\mathrm{d} P}{\mathrm{~d} x}\right] \mathrm{d} x\right],
\end{aligned}
$$

where $P_{1}$ is the applied pressure and $P_{2}$ is the pressure at the top of the engagement length, $u(x)$ is the piston deviation, $U(x)$ is the cylinder deviation, $h_{o}$ is the initial clearance between piston-cylinder unit and $r_{p_{o}}$ is the undistorted radius of the piston [16].

Figure 4 shows a view of piston-cylinder clearance; with indication of radial changes between the piston and cylinder in their engagement length (defined as the coupling length of piston and cylinder between $x=0$ and $x=1$ ). Under absolute conditions, $P_{2}=P_{o}$ and in gauge condition $P_{2}=$ $P_{\text {atm. }}$. Appropriate diameter irregularities are emphasized and reference is made to the radius.

For an undeformed piston-cylinder unit or in the case of perfect geometry, i.e. uniform clearance or constant $h$ and $u=$ $U=0$, i.e. no piston or cylinder deviation, the effective area $A_{o}$ is exactly the arithmetic mean between the areas of the piston and cylinder. From equation (4)

$$
\begin{gathered}
A_{o}=\pi r_{p_{o}}^{2}\left(1+\frac{h_{o}}{r_{p_{o}}}\right) . \\
\text { Also } A_{o}=\frac{\pi r_{p 0}^{2}+\pi r_{c 0}^{2}}{2}=\frac{\pi r_{p o}^{2}+\pi\left(r_{p o}+h_{o}\right)^{2}}{2}
\end{gathered}
$$




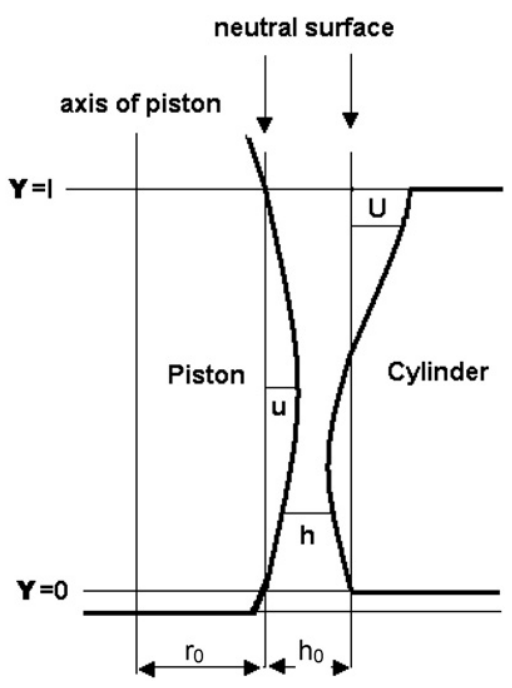

Figure 4. Schematic of piston-cylinder unit with indication of radial changes between the piston and the cylinder in their engagement length (defined as the coupling length of piston and cylinder between $x=0$ and $x=1$ ). Absolute condition: $p_{2}=p_{o}$. Gauge condition: $p_{2}=p_{\text {atm }}$.

where $r_{c_{o}}=r_{p_{o}}+h_{o}$ is the initial inner radius of the cylinder. By performing simple mathematical calculation under the condition that the initial clearance $h_{o} \ll 1$, it can be seen that equations (5) and (6) are the same.

Although the pressure transmitting medium in our case is nitrogen gas, however, since the gap is lubricated by oil, the solution of the Navier-Stokes equation and the equation of continuity give the relation between the pressure distribution along the gap length, the gap profile $h(x)$ and the properties of the liquid and is given as [17]

$$
P(x)=P_{1}+K \int_{0}^{x} \frac{\eta(P)}{\rho(P)} \frac{1}{h^{3}(x)} \mathrm{d} x,
$$

where $P$ denotes the initial pressure. Since the density, viscosity and $K$ depend upon $P(x)$, the pressure distribution in the gap profile is calculated using Navier-Stokes equation.

At ambient $P(x)=P_{2}$, i.e. atmospheric pressure; hence

$$
P_{2}=P_{1}+K \int_{0}^{l} \frac{\eta(P)}{\rho(P)} \frac{1}{h^{3}(x)} \mathrm{d} x
$$

and

$$
K=-\left(P_{1}-P_{2}\right) / \int_{0}^{l} \frac{\eta(P)}{\rho(P)} \frac{1}{h^{3}(x)} \mathrm{d} x .
$$

Further, since di-ethyl-hexyl-sebacate oil is used as lubricating oil in the clearance between the piston and the cylinder, the density $\rho$ and the dynamic viscosity $\eta$, dependent on pressure (in $\mathrm{MPa}$ ) at $20^{\circ} \mathrm{C}$, can be described by the following equations [18]:

$$
\begin{aligned}
\rho= & 912.6657+0.752 P-1.645 \times 10^{-3} \cdot P^{2} \\
& -1.456 \times 10^{-6} \cdot P^{3}\left(\mathrm{~kg} \mathrm{~m}^{-3}\right)
\end{aligned}
$$

and

$$
\eta=0.021554\left(1+1.9004 \times 10^{-3} \cdot P\right)^{8.8101}(\mathrm{~Pa} \mathrm{~s}) .
$$

In the first iteration, the pressure in the gap was taken to be linearly changing from $P_{1}$ to ambient and the distortion calculated. For this pressure distribution, the coefficient $K$ was established with equation (8). Subsequently, using equation (7) again, a new pressure distribution was calculated and a new $K$ determined. In this way the FEM calculations were iterated with each of the revised profiles and the process was continued till convergence in the pressure distribution was achieved.

The theoretical estimations have been carried out using the following calculations:

For theoretical estimations, the distortions in piston and cylinder have been estimated using the corresponding distortion terms from the Johnson-Newhall equation. For a simple piston-cylinder unit, Johnson and Newhall as well as Tsiklis [15, 19], demonstrated the distortion coefficient under conditions of no end loading or no axial loading, and a constant pressure profile assumed as $P_{1} / 2$ is given as

$$
\lambda=\frac{3 v_{p}-1}{2 E_{p}}+\frac{1}{2 E_{c}}\left[\frac{R_{c}^{2}+r_{c_{o}}^{2}}{R_{c}^{2}-r_{c_{o}}^{2}}+v_{c}\right],
$$

where the first term is the contribution to the distortion of the piston and the second to that of the cylinder. In equation (9), $v_{p}$ and $v_{c}$ are the values of Poisson's ratio, $R_{c}$ is the outer radius of the cylinder and $r_{c_{o}}$ is the inner radius of the cylinder, $E_{p}$ and $E_{c}$ are Young's modulus of elasticity of piston and cylinder respectively. For the theoretical calculation of piston and cylinder deviation and hence the clearance, we have used equations (3) and (9) in the following manner [20]:

$$
h(x)=h_{o}+U(x)-u(x)=r_{c}-r_{p} .
$$

Assuming that equation (9) is valid for local axial variations in radial pressure loading, the piston's deviation is given as the difference in the initial radius of the piston and the radius of the piston after deviation. Using equations (3) and (9), we obtain

$u(x)=r_{p}-r_{p_{o}}=r_{p_{o}}\left\{\left[1+\left(\frac{3 v_{p}-1}{2 E_{p}}\right) P(x)\right]^{1 / 2}-1\right\}$.

Similarly for the cylinder, its deviation is given as the difference in the radius of the cylinder after deviation and the initial radius of the cylinder:

$U(x)=r_{c}-r_{c_{o}}$

$=r_{c_{o}}\left[\left\{1+\frac{1}{2 E_{c}}\left(\frac{R_{c}^{2}+r_{c_{o}}^{2}}{R_{c}^{2}-r_{c_{o}}^{2}}+v_{c}\right) P(x)\right\}^{1 / 2}-1\right]$.

Hence, using $P(x)$ from equation (7), $u(x)$, and $U(x)$ have been estimated and we have calculated $A_{p}$ for all applied pressures (1-8 MPa) using equation (4) for theoretical calculations.

In simulation calculations we have calculated $A_{p}$ using equation (4) again and for this we have used the data of elastic-strain obtained after FEM analysis using ANSYS. As mentioned earlier, for the estimation of strain a linear pressure variation along the engagement length has been initially assumed, and the obtained distortion data and gap profile are used in the Navier-Stokes equation and the results iterated till convergence is achieved. Consequently the $\mathrm{d} p / \mathrm{d} x$ is calculated using Navier-Stokes equation. From the converged values 


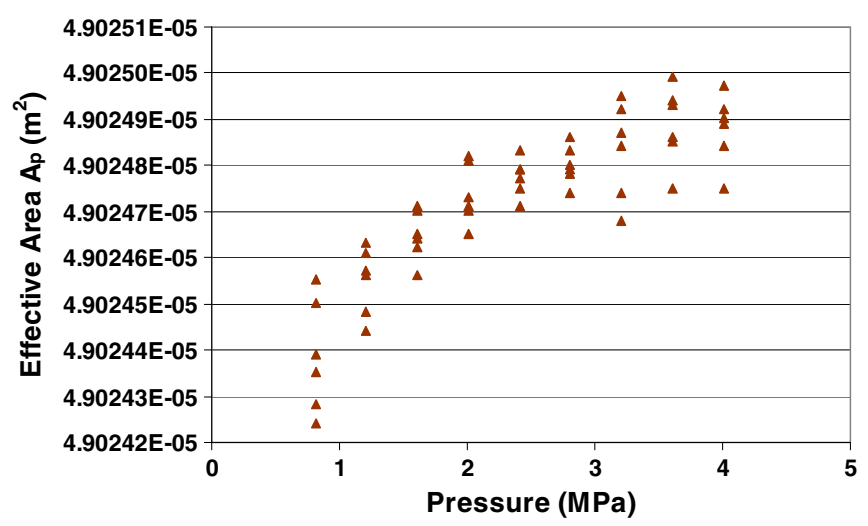

Figure 5. The experimentally obtained cross-float data of effective area variation as a function of pressure.

of strain, $u(x)$ and $U(x)$ have been estimated. $A_{p}$ has been estimated from equation (4) using the value of $\mathrm{d} p / \mathrm{d} x$ calculated from equation (7). These results have been used to obtain $A_{p}$ as a function of pressure and the calculated results are compared with the actual experimentally obtained values.

\section{Results and discussion}

\subsection{Experimental results}

Figure 5 shows the variation of experimentally obtained effective area with pressure up to $4 \mathrm{MPa}$. The obtained data were subjected to linear regression and the equation obtained yields

$$
A_{p}=4.9024467 \times 10^{-5}+1.1409144 \times 10^{-10} P_{1} .
$$

From the above fitting equation, the zero pressure effective area $A_{0}$ and the distortion coefficient were calculated and found to be $4.9024467 \times 10^{-6} \mathrm{~m}^{2}$ at $20{ }^{\circ} \mathrm{C}$ and $2.327 \times$ $10^{-6} \mathrm{MPa}^{-1}$ respectively. The uncertainties in the effective area and the distortion coefficient have been estimated to be $3.36367 \times 10^{-11}$ and $2.46 \times 10^{-7}$ respectively. The relative expanded uncertainty in pressure measurement estimated as per ISO and NABL guidelines has been estimated as $26 \times$ $10^{-6}$ at $k=2$.

\subsection{Simulation and theoretical calculations}

As mentioned earlier, the pressure distribution along the engagement length of piston and cylinder has been calculated using Navier-Stokes equation. The obtained pressure profiles for a few studied pressures are plotted in figure 6 . The obtained profiles are found to be almost linear in nature all along the engagement length for each of the pressures studied. The pressure distribution is a direct reflection of the fact that at the top of the clearance the pressure falls to atmospheric pressure while the designated pressure is applied at the origin or the base of the piston.

For the simulation using FEM, the full view of the deformed portion of piston-cylinder assembly as simulated in FDM at a pressure of $8 \mathrm{MPa}$ has already been shown in figure $3(a)$, and the zoomed portion of the same is shown

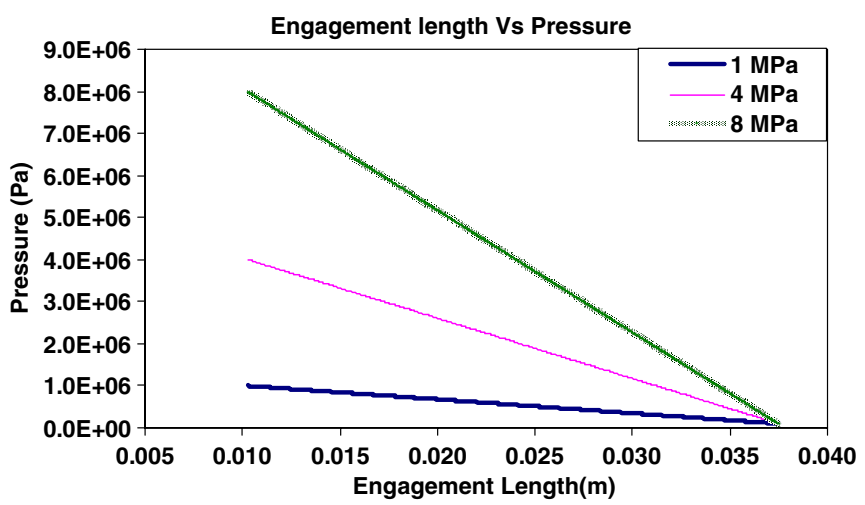

Figure 6. A representative depiction of the applied pressure profile over the engagement length at a few studied pressures.

in figure $3(b)$. The contour plot of the pc assembly for FDM is shown in figure 7 . It is seen that, as a result of pressure distribution, a gap profile is created within the engagement length, the magnitude of which is a function of the applied pressure. The load on the piston and the cylinder creates a force acting along the $x$-axis which leads to a local longitudinal compression and, consequently, to a local crossexpansion of the cylinder. This was confirmed by calculations performed both in the presence and absence of the load on the cylinder. Also the top of the cylinder is kept constrained which is a necessary assumption for simulation [13]. The deformed assembly shows an increased gap at the bottom of the assembly. This gap was again found to be dependent upon the pressure applied and increases linearly with increase in applied pressure. The representative gap width variations obtained from FEM analysis are depicted in figure 8 for a few studied pressures. The gap width at the bottom of the piston is typically about $0.691 \mu \mathrm{m}$ at $1 \mathrm{MPa}$ and about $0.803 \mu \mathrm{m}$ at a pressure of $8 \mathrm{MPa}$. This gap is seen to decrease at the top of the piston due to linear decrease in pressure as already discussed. Further, as the applied pressure increases, the gap width at the top of the engagement length also increases slightly as compared to the gap at ambient.

The radial distortion of the piston and the cylinder as a function of normalized engagement length is shown in figure 9 for pressures $8 \mathrm{MPa}$ and $1 \mathrm{MPa}$ for piston and cylinder. The graph shows the relative distortion in the piston and cylinder at 1 and $8 \mathrm{MPa}$ with respect to the radial clearance at zero pressure. As already observed in figure 8, the distortion is maximum at the bottom of the piston and cylinder where the maximum pressures act while it decreases toward the top of the piston-cylinder assembly. It is significant to note that the cylinder undergoes higher distortion compared to the piston. Also, although the cylinder distorts predominantly in one direction, the distortion in the piston reverses slightly toward the top of the engagement length.

In comparison, the gap width as well as the radial distortion as calculated using Dadson's and Newhall's equations, shown in figures 10 and 11 respectively, shows a few variations. The gap width again shows an increase at the bottom with increase in applied pressure although the magnitude of the gap is seen to be lower as compared to the 


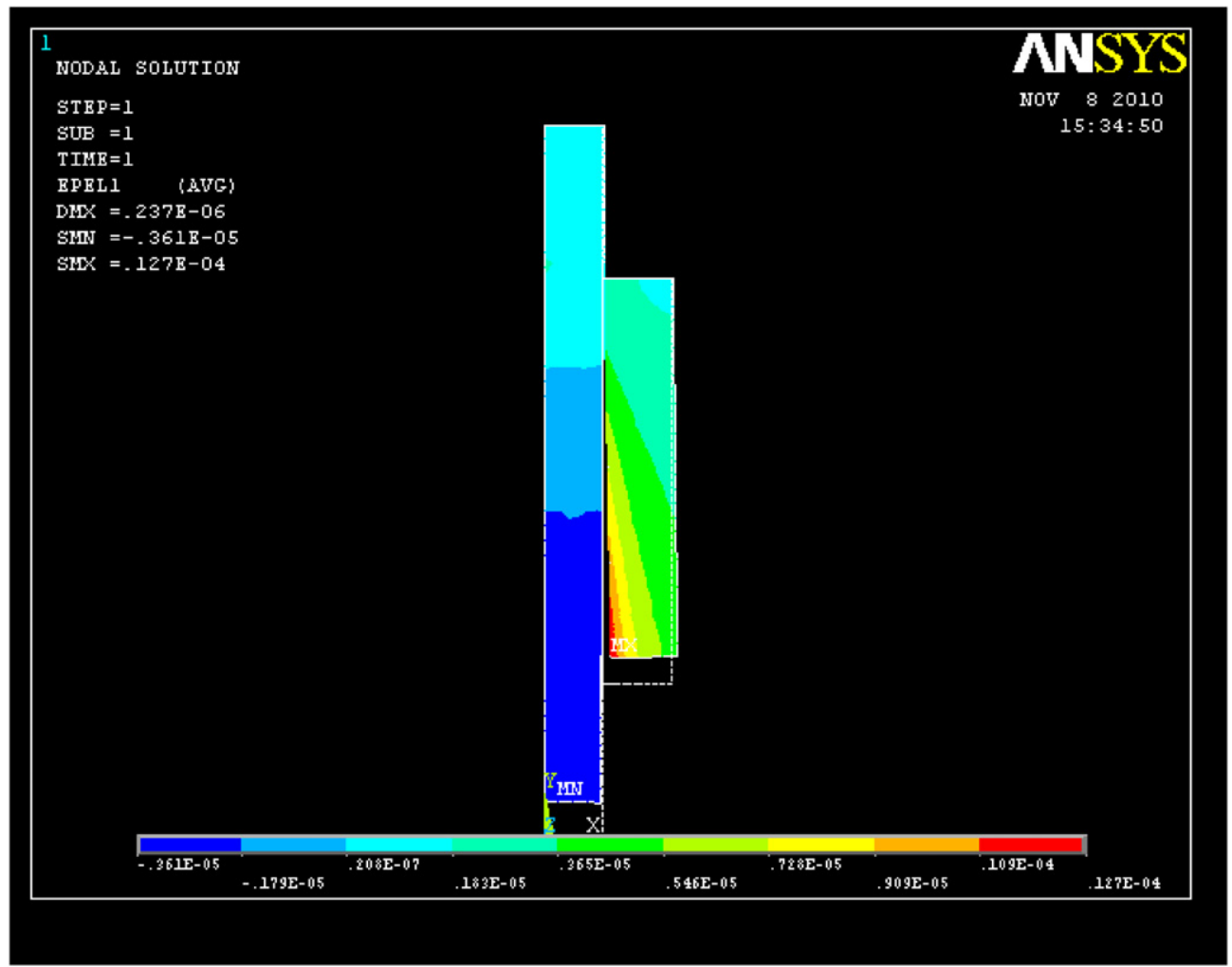

Figure 7. Image of the distorted piston-cylinder assembly after ANSYS simulation in FDM.

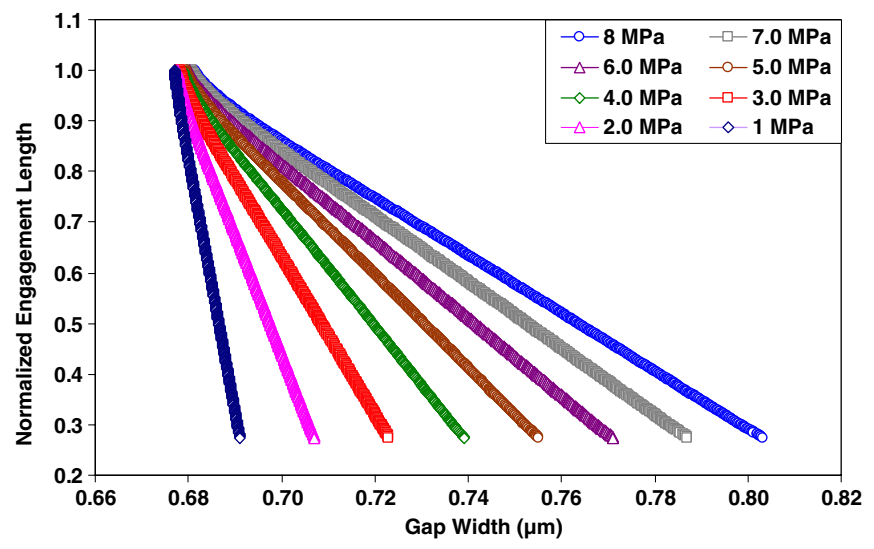

Figure 8. Gap profile of the pc assembly as obtained from FEM simulation.

estimation from FEM analysis. It may be noted that the slope in the gap variation increases with increase in pressure and the radial distortion shows distortion in one direction only for all applied pressures. In addition, the gap width at the top of the engagement length does not show an appreciable variation with increase in applied pressure as was observed in the case of FEM calculations. Likewise the magnitude of the radial distortion is also comparatively lower in this case and the distortion is unidirectional for piston as well as cylinder.

The comparison between theoretical and simulated $A_{p}$, calculated using equation (4), as well as experimentally obtained $A_{p}$ using the cross-floating technique, is shown in figure 12. The experimental points were calculated from the

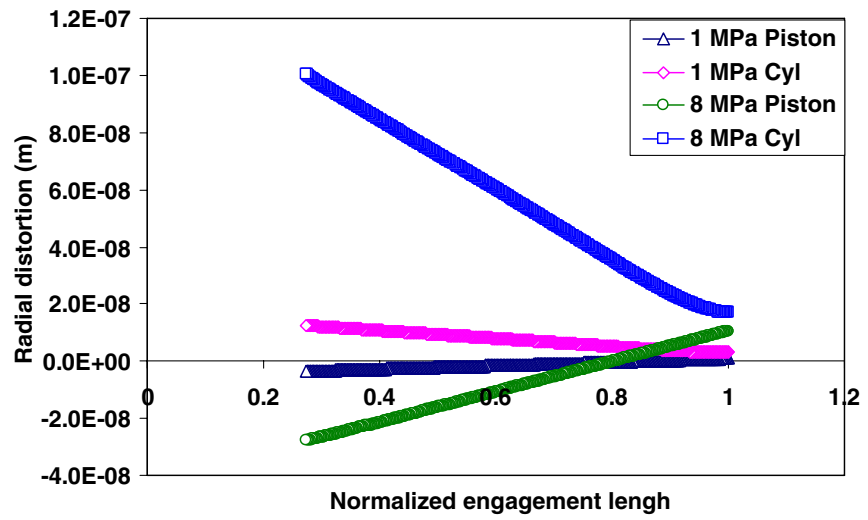

Figure 9. Radial distortion of the piston and cylinder at pressures of 1 and $8 \mathrm{MPa}$ as obtained from FEM simulation.

regression equation (13) obtained from fitting the experimental data and calculating $A_{p}$ as a function of pressure up to $8 \mathrm{MPa}$. The calculated experimental points have been extrapolated to $8 \mathrm{MPa}$ for comparison purposes. For reference purposes, the average effective area obtained experimentally at each pressure point, up to a pressure of $4 \mathrm{MPa}$, is also plotted alongside. It may be pointed out here that we have recently established our CMCs up to $40 \mathrm{MPa}$ gas pressures with our traceability starting from UIM with very low pressures [21]. The pistons with various pressure ranges have been calibrated in overlapping pressure ranges and the traceability extended to the piston with next higher pressures. Likewise, in the present case, the fitted linear equation has been used to generate points up to $8 \mathrm{MPa}$ for comparison purposes only. 


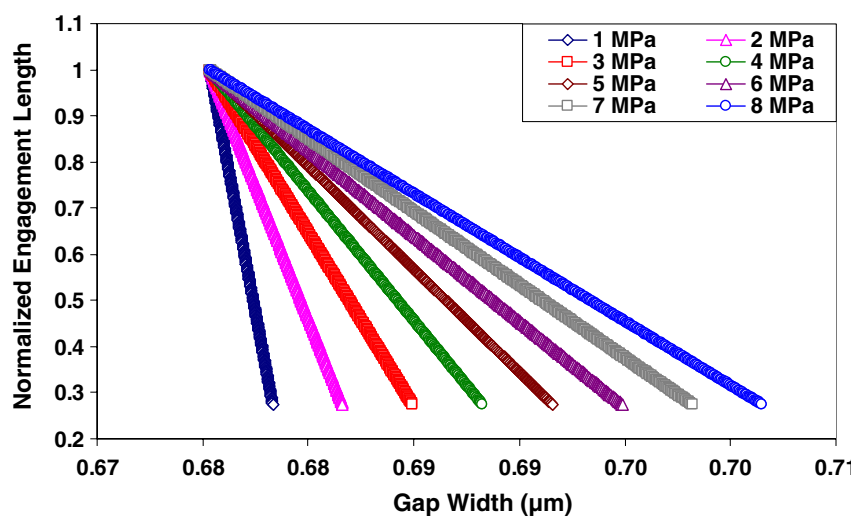

Figure 10. Gap profile of the piston-cylinder assembly as obtained from theoretical estimations.

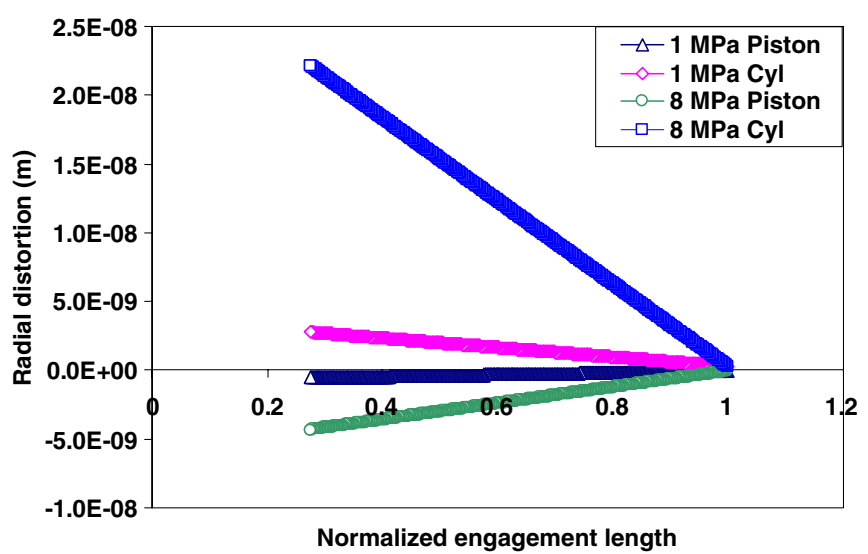

Figure 11. Radial distortion of the piston and cylinder obtained from theoretical estimations.

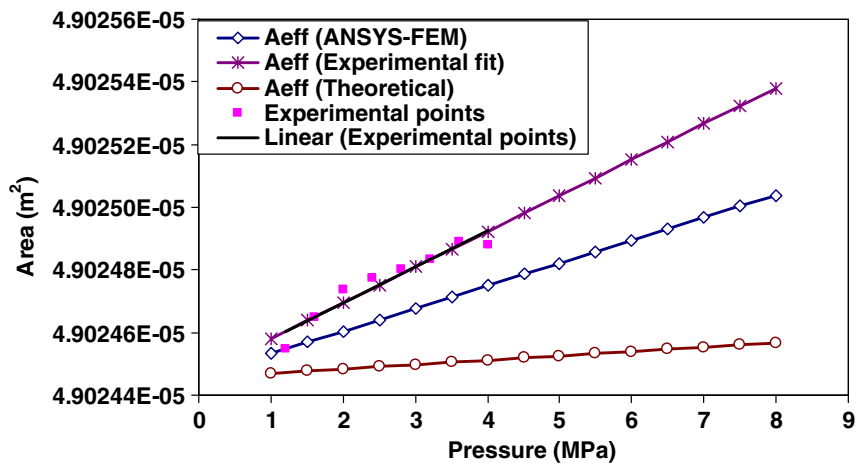

Figure 12. A comparative graph of $A_{p}$ versus pressure between experimental data and simulated as well as theoretical estimations.

As expected, the $A_{p}$ values are seen to be increasing as the pressure is increased both in theoretical and simulated results. As compared to our actual experimentally obtained values, the $A_{p}$ values using FEM calculations show better agreement and agree within about $3.5 \times 10^{-6}$ at $4 \mathrm{MPa}$ and $7 \times 10^{-6}$ at a pressure of $8 \mathrm{MPa}$. The theoretical estimation is found to be in worse agreement with the experimental results and varied by about $17 \times 10^{-6}$ at $8 \mathrm{MPa}$.

The results thus obtained were used to estimate the distortion coefficient $\lambda$, and the obtained variation of $\lambda$ with

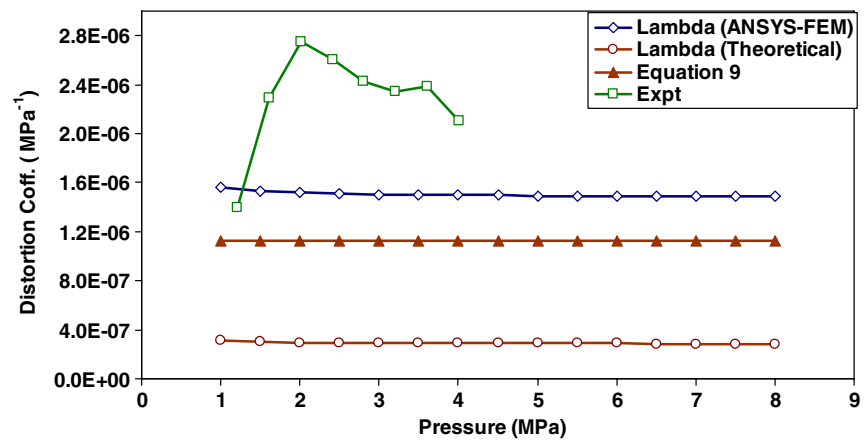

Figure 13. Comparative graph of distortion coefficient variation as a function of pressure obtained from various calculation approaches.

pressure is shown in figure 13. Once again the trend of the differences obtained between various approaches to estimation are reflected in a similar manner. The $\lambda$ values calculated using pressure profile in FEM calculations show the values closest to the experimentally obtained values, while the theoretical estimations show values lower by an order. As the pressure increases from 1 to $8 \mathrm{MPa}$, the distortion co-efficient is seen to remain almost constant with a slightly higher value toward the lower pressure end. Similar observations have been reported by Buonanno et al [22]. For FEM calculations, $\lambda$ varies from a value of $1.56 \times 10^{-6} \mathrm{MPa}^{-1}$ at $1 \mathrm{MPa}$ to $1.49 \times$ $10^{-6} \mathrm{MPa}^{-1}$ at a pressure of $8 \mathrm{MPa}$. The experimentally obtained distortion coefficient from regression analysis, has been found to be $2.327 \times 10^{-6} \mathrm{MPa}^{-1}$. Further, the distortion coefficient was also calculated directly using equation (9) and is also plotted in figure 13. These values do not involve the pressure profile and hence are plotted as a constant value. It is clear that these values are close to the values estimated using FEM and show significant difference from the theoretically estimated values. Hence the FEM simulated results are found to be in closer agreement with the expected results.

Further, as mentioned earlier, the FEM simulations were repeated with smaller as well as larger mesh size of the modeled finite element structure. As already pointed out, the simulations were repeated for mesh sizes 3 and 5 apart from the size 4 results mentioned above. The results obtained are also mentioned in table 3 . From table 3 , it can be clearly seen that there is absolutely no change in the values obtained for $A_{0}$, although a negligible change in average distortion coefficient value is seen. Likewise, the simulations were also done for an assumed linear pressure variation in the gap, and total neglect of the presence of lubricating oil in the gap, and once again, the results were found to be exactly the same.

In a nutshell, from the results obtained the following points are made clear.

(1) The consequence of almost linear pressure variation across the engagement length leads to a gap width and radial distortion profile which qualitatively differs from the theoretically estimated profile at the top of the engagement length. The theoretical profile shows continuous increase in the gap at the bottom of the piston with increase in pressure as compared to the gap at ambient, and the gap at the top does not show a significant 
Table 3. A comparative summary of the results obtained from various calculations.

\begin{tabular}{|c|c|c|c|c|c|c|}
\hline Parameter & $\begin{array}{l}\text { Experimentally } \\
\text { obtained }\end{array}$ & $\begin{array}{l}\text { Dimension } \\
\text { measurements }\end{array}$ & $\begin{array}{l}\text { FEM mesh } \\
\text { size } 4\end{array}$ & $\begin{array}{l}\text { FEM mesh } \\
\text { size } 3\end{array}$ & $\begin{array}{l}\text { FEM mesh } \\
\text { size } 5\end{array}$ & $\begin{array}{l}\text { Theoretical } \\
\text { calculations }\end{array}$ \\
\hline$A_{0}\left(\times 10^{-6} \mathrm{~m}^{2}\right)$ & 4.9024467 & 4.902445586 & 4.9024460 & 4.9024460 & 4.9024460 & 4.9024457 \\
\hline Agreement with experimental $A_{0}$ & - & $0.23 \times 10^{-6}$ & $0.14 \times 10^{-6}$ & $0.14 \times 10^{-6}$ & $0.14 \times 10^{-6}$ & $0.20 \times 10^{-6}$ \\
\hline$\lambda\left(\times 10^{-6} \mathrm{MPa}^{-1}\right)$ & 2.327 & - & (avg.) 1.5036 & (avg.) 1.5040 & (avg.) 1.5049 & (avg.) 0.291 \\
\hline
\end{tabular}

change. However, the FEM estimated radial distortion and gap profiles show that there is a significant increase in the gap at the top of the engagement length with increase in pressure. This may be a manifestation of the fact that in FEM calculations, the magnitude of the distortion is higher for both the piston and the cylinder as compared to the theoretical results and hence the observation.

(2) Owing to larger distortion, the distortion coefficient is found to be higher in FEM results, which actually shows better agreement with the experimentally obtained values. It may be added here that the experimentally obtained distortion coefficient also includes a reflection of the $\lambda$ value of the reference standard and hence may show higher or lower values depending upon the $\lambda$ value of the reference standard.

(3) The $u(x)$ and $U(x)$ values in theoretical estimations have been calculated using the $\lambda$ values, which in turn have been calculated from the material properties of the piston and cylinder. However, it has been reported that for the use of equation (9) at pressures higher than $0.5 \mathrm{MPa}$, the uncertainties in the estimations of $\lambda$ can be of the order of 100-200\% [1], which in turn would affect the estimation of $u(x)$ and $U(x)$ and ultimately $A_{p}$ and the distortion profiles. This explains the large deviation of the theoretically estimated results from the experimental as well as FEM results. In fact it has been reported that for pressures higher than $0.5 \mathrm{MPa}$, more reliable values for the distortion coefficient would be achieved by using comparison and experimental measurements as well as similarity method [1].

(4) Another factor affecting the difference obtained for $\lambda$ may include the assumptions under which equation (9) has been proposed. The equation is valid for no end-loading of the cylinder and a constant mean pressure value of $P_{1} / 2$ in the clearance. Therefore, the $\lambda$ values are found to be lower in the case of theoretical calculations, and hence the corresponding $u(x)$ and $U(x)$ using equations (11) and (12) respectively are lower as already depicted by the radial distortion profile in figure 11 . Therefore, the resulting values obtained for $A_{p}$ are found to be lower as compared to the simulated and experimental results. This could be one of the reasons for the difference observed in FEM and theoretical estimations despite using the same properties of the materials in both approaches.

(5) Although the experimentally fitted values are extrapolated up to a pressure of $8 \mathrm{MPa}$ where they show an agreement of $7 \times 10^{-6}$ with the FEM results, at the actual highest experimental pressure of $4 \mathrm{MPa}$, the agreement is found to be $3.5 \times 10^{-6}$ which is much better compared to the extrapolated data at $8 \mathrm{MPa}$.

(6) The differences in the experimental and simulated results could also come about due to the starting values used in FEM, obtained from dimension measurements. As mentioned earlier, the uncertainty in dimension measurements is approximately $\pm 0.5 \mu \mathrm{m}$ at $k=2$ for the values of the radii used. The estimated uncertainty is close to the initial gap of $0.675 \mu \mathrm{m}$. Hence the actual gap may be higher or lower than the value used and hence lead to the difference obtained in experimental and simulated results. However, since the agreement is within $3.5 \times$ $10^{-6}$ at a pressure of $4 \mathrm{MPa}$, the results are well within the uncertainty of our own experimental results.

Further simulations with variation in gap width as well as the shape of the piston-cylinder assembly are under progress.

\section{Conclusions}

A numerical methodology based on FEM has been used to study the gap profile, radial distortion, pressure distortion coefficient and effective area in FDM for a pneumatic $8 \mathrm{MPa}$ piston-cylinder assembly. The effective area and pressure distortion coefficients were also calculated theoretically using Dadson's model and compared with the simulated and the experimentally obtained results. The simulation calculations were carried out for gauge pressure ranging from 1 to $8 \mathrm{MPa}$ using the Finite Element software ANSYS 9.0. The effective area variation with pressure was found to agree within $7 \times$ $10^{-6}$ at a pressure of $8 \mathrm{MPa}$ with the experimentally obtained values while the theoretical calculations vary by about $17 \times$ $10^{-6}$.

The gap profile and radial clearance profiles show increased gap and therefore strain, at the bottom of the piston-cylinder assembly which decreases toward the top due to pressure distribution in the gap profile. The distortion coefficient from simulation studies shows better agreement with experiment compared to the theoretically obtained values.

\section{References}

[1] Molinar G F and Pavese F 1992 Modern Gas-Based Temperature and Pressure Measurements (New York: Plenum)

[2] Sabuga W, Molinar G, Buonanno G, Esward T, Legras J C and Yagmur L 2006 Finite element method used for calculation of the distortion coefficient and associated uncertainty of PTB 1 GPa pressure balance-EUROMET project 463 Metrologia 43 311-25 
[3] Molinar G, Sabuga W, Robinson G and Kegras J C 1998 Comparison of methods for calculating distortion in pressure balances up to $400 \mathrm{MPa}$-EUROMET project 256 Metrologia 35 739-59

[4] Dadson R S, Lewis S L and Peggs G N 1982 The Pressure Balance: Theory and Practice (London: HMSO)

[5] Peggs G N 1977 A method for computing the effective area of a pressure balance from diametrical measurements National Physical Laboratory Divisional Report MOM 23, Teddington, UK

[6] Driver R G, Olson D A, Dilawar Sharma N and Bandyopadhyay A K 2007 Final report on key comparison APMP.SIM.M.P.-K1c: bilateral comparison between NIST (USA) and NPLI (India) in the pneumatic pressure region 0.4 MPa-4.0 MPa Metrologia 4407002

[7] Bandyopadhyay A K et al 2003 Results of the APMP pressure key comparison APMP.M.P-K1c in gas media and gauge mode from 0.4-4 MPa Metrologia 4007002

[8] Sharma J K N, Jain K K, Bandyopadhyay A K and Molinar G F 1989 Intercomparison of pneumatic differential pressure measurements at $30-150 \mathrm{kPa}$ at high line pressures up to 7.5 MPa High Temp. High Press. 21727

[9] Chaudhary K P, Sharma R, Farid N and Singhal R P 2002 Calibration of step gauge by substitution method on coordinate measuring machine and evaluation of uncertainty of measurement MAPAN 1767

[10] Sharma J K N, Jain K K, Bandyopadhyay A K and Jager J 1988 International intercomparison of pressure standards in the pneumatic pressure region 0.4-4.0 MPa between NPL (India) and PTB (FRG) J. Phys. E: Sci. Instrum. 21635

[11] Dilawar Sharma N, Vijay Kumar A, Sharma D R and Bandyopadhyay A K 2009 Characterization of pneumatic piston gauge through continuous chain of traceability from very low gas pressures 7 th Int. Conf. on
Advances in Metrology (ADMET-2009 (009) (National Physical Laboratory, New Delhi)

[12] NABL April 2000 Guidelines for estimation and expression of uncertainty in measurement Department of Science and technology, New Delhi NABL-141

[13] Sabuga W 1995 Elastic distortion calculation at PTB on LNE $200 \mathrm{MPa}$ balances EUROMET project 256 PTB-W-63 Report Physikalische-Technische Bundesanstalt, Braunschweig, Germany

[14] Esward T J, Preston R C and Gelat P N 2003 Piston-cylinder pressure balance design with a negligible distortion coefficient Measurement 14 796-806

[15] Johnson D P and Newhall D H 1953 The piston gauge as precise pressure-measuring instrument Trans. ASME 75301

[16] Yagmur L and Bagli E F 2009 Experimental and dimensional characterization of a prototype piston cylinder unit and validation using finite element analysis Measurement 42678

[17] Molinar G F, Maghenzani R, Cresto P C and Bianchi L 1992 Elastic distortions in piston-cylinder units at pressures up to $0.5 \mathrm{GPa}$ Metrologia 29425

[18] Vergne Ph 1990 High Temp. High Press. 22613

[19] Tsiklis D S 1968 Handbook of techniques High Pressure Research and Engineering (New York: Plenum)

[20] Buonanno G, Giovinco G, Molinar Min Beciet G, Delajoud P and Haines R 2006 A FEM analysis of a negative free deformation pressure balance operating up to $100 \mathrm{MPa} X V I I$ IMEKO World Congress Metrology for Sustainable Development (Rio de Janeiro, Brazil)

[21] Complete CMCs in Mass and related quantities for NPL India http://www.bipm.org/

[22] Buonanno G, Dell'Isola M and Maghenzani R 1999 Finite element analysis of pressure distortion coefficient and piston fall rate in a simple pressure balance Metrologia 36579 